\title{
Essais
}

ESSAIS

Revue interdisciplinaire d'Humanités

\section{Réflexions autour de la philosophie de l'environnement}

Entretien avec Catherine Larrère

Fabien Colombo, Nestor Engone Elloué et Bertrand Guest

\section{(2) OpenEdition}

\section{Journals}

Édition électronique

URL : http://journals.openedition.org/essais/533

DOI : 10.4000/essais.533

ISSN : 2276-0970

\section{Éditeur}

École doctorale Montaigne Humanités

Édition imprimée

Date de publication : 1 janvier 2018

Pagination : 135-151

ISBN : 979-10-97024-03-1

ISSN : 2417-4211

\section{Référence électronique}

Fabien Colombo, Nestor Engone Elloué et Bertrand Guest, « Réflexions autour de la philosophie de l'environnement », Essais [En ligne], 13 | 2018, mis en ligne le 01 décembre 2019, consulté le 10 décembre 2020. URL : http://journals.openedition.org/essais/533 ; DOI : https://doi.org/10.4000/ essais. 533 


\section{Réflexions autour de la philosophie de l'environnement Entretien avec Catherine Larrère ${ }^{1}$}

\section{Bertrand Guest, Fabien Colombo, Nestor Engone Elloué}

Catherine Larrère est Professeure émérite à l'Université de Paris IPanthéon-Sorbonne. Spécialiste de philosophie morale et politique, elle a particulièrement contribué à introduire la philosophie de l'environnement en France. Elle a notamment publié Les philosophies de l'environnement (Paris, PUF-collection Philosophies, 997), et en collaboration avec Raphaël Larrère, Du bon usage de la nature, Pour une philosophie de l'environnement (Paris, Aubier, 1997) et Penser et Agir avec la Nature (Paris, La Découverte, 2015)².

Nestor Engone Elloué (N.E.E.) : Le débat en philosophie environnementale s'est longtemps articulé autour des enjeux éthiques. Quel est votre avis à propos de la question du choix entre une éthique du respect de la nature et une éthique de la responsabilité ?

Catherine Larrère (C.L.) : Au début ces éthiques se sont développées de façon indépendante, chacune ignorant l'autre. Il y a effectivement des éthiques du respect de la nature qui se sont développées davantage dans les pays anglophones (Amérique du nord, Australie et Angleterre), alors qu'en Europe continentale, la réflexion s'est faite beaucoup plus autour des questions de responsabilité et donc autour d'un rapport à la technique, parce qu'en Europe, la référence à la nature n'est pas du tout unifiante. Ce n'est pas qu'il n'y en a pas, c'est juste qu'elle n'est pas unifiante, alors que la technique fait partie de l'identité européenne. En fait, dans ces éthiques qui sont une sorte de réflexion critique (et qui ont des dimensions identitaires), les États-Unis ont réfléchi à partir de leur rapport à la nature et l'Europe a réfléchi sur son rapport à la technique. Et pendant longtemps chacun a ignoré l'autre. C'est même étonnant : Hans Jonas dans le Principe Responsabilité (1979) fait un

Entretien réalisé le 8 avril 2015 à Bordeaux.

2 Ouvrage en cours de parution au moment où se tenait cet entretien. 
éloge appuyé du mode américain de protection de la nature en wilderness, mais ne va pas plus loin. À ce moment-là, il n'a pas lu l'éthique environnementale. Et quand j'ai rencontré Callicott ${ }^{3}$, je lui ai demandé s'il avait lu Jonas, il m'a gentiment dit qu'il allait le lire, mais ne l'a jamais lu. Simplement, avec la globalisation, c'est-à-dire avec la transfusion du naturel et de l'humain, il n'y a plus à choisir entre les deux. Et de toute façon ce n'est pas exclusif, " Respect " et "Responsabilité ", c'est plutôt une question d'accent. Mais il est certain que ce n'est plus possible d'isoler une éthique de protection de la nature et une éthique de responsabilité vis-à-vis des conséquences de nos actions techniques. Les deux sont liées et il faut les prendre en compte.

Bertrand Guest (B.G.) : La distinction entre philosophie de la technique et philosophie de la nature est interrogée dans votre travail autour de la préoccupation d'une connaissance physique et morale à la fois. Peut-être qu'il n'y a plus de distinction justifiable entre une philosophie de la technique d'un côté et une philosophie de la nature de l'autre, d'autant plus que se développent des branches interdisciplinaires de digital humanities d'un côté, d'environmental humanities de l'autre. Est-ce que ces deux façons de faire des "humanités », les humanités environnementales et les humanités digitales, sont pour vous en opposition?

C.L. : Qu'entendez-vous par humanités digitales?

B.G. : L'étude des nouvelles technologies et des manières de lire renouvelées qu'elles induisent, les manières de transmettre les textes, de les traduire, de les comprendre.

C.L. : Je vous pose cette question parce que j'entendais deux interrogations dans votre question, l'une sur le lien entre philosophie de la nature et philosophie de la technique, l'autre sur le digital. Sur la première, c'est la grande différence entre $D u$ bon usage de la nature $e^{4}$ et le livre que nous sortons à la fin du mois, Penser et agir avec la nature ${ }^{5}$ dans lequel se trouve une véritable philosophie de la technique. Du bon usage de la nature n'en comportait pas, restant à l'idée que la technique c'est de la science appliquée, or ce nouveau livre y consacre une partie entière. Le problème rapidement résumé est que vous pouvez toujours aller voir chez Platon et Aristote, il n'est pas véritablement question de "technique». Ça s'appelle autrement. Dans ce qu'ils disent sur l'art, au sens d'" artisanat ", il n'y a pas à proprement parler de philosophie de la technique. Le terme de "philosophie de la technique " apparait en Allemagne au XIX ${ }^{\mathrm{e}}$ siècle et l'on se met à en parler quand la technique cesse d'être un intermédiaire entre l'homme et la nature et qu'on se pose la question

3 John Baird Callicott est un philosophe américain, spécialiste d'éthique environnementale.

4 Catherine et Raphaël Larrère, Du bon usage de la nature. Pour Une philosophie de l'environnement, Paris, Aubier, 1997.

5 Catherine Larrère, Raphäl Larrère, Penser et agir avec la nature. Une enquête philosophique, Paris, La Découverte, coll. «Sciences humaines », 2015. 
du rapport de l'homme à ses objets techniques. Donc au moment où la technique devient un intérêt philosophique elle perd son rapport avec la nature. Disons qu'en réfléchissant sur la nature on ne tombe pas sur la technique, et en réfléchissant sur la technique on peut ne pas rencontrer la nature. Si vous lisez André Leroi-Gourhan ou Simondon, il est question de s'interroger sur les rapports ; de savoir si la technique est une extériorisation de l'homme; d'interroger ce qu'est qu'un objet technique, etc. La nature, il n'en est pas question. Sur la question du digital comme vous dites, je n'ai pas grand-chose à dire. Il se trouve que je fais partie du Comité d'éthique Cirad-Inra, et actuellement nous sommes en train d'élaborer un rapport non pas sur les Big Data, mais sur la multiplication des données, qui d'après ce que je vois, pose des problèmes de propriété et d'économie de notre connaissance, plus que d'écologie.

Fabien Colombo (F.C.) : Concernant précisément l'impact de la technique humaine sur la nature, en novembre prochain vous organisez un événement au Collège de France avec Philippe Descola sur le thème de l'Anthropocène. Pouvez-vous nous expliquer en quoi cette notion marque un tournant pour les approches de la philosophie environnementale?

C.L. : Michel Serres est sans doute un des premiers à avoir dit très clairement dans le Contrat Naturel ${ }^{6}$ que la globalisation de l'environnement marque un point nouveau qui est celui de l'impossibilité (vous parliez d'humanities), de la séparation classique entre ce qui relève de la nature et ce qui relève de l'homme. Michel Serres le dit avec son style particulier, en substance "l'histoire de l'homme rentre dans l'histoire naturelle et l'histoire naturelle rentre dans l'histoire de l'homme ». L'Anthropocène est le nom qui est donné à cette fin de la séparation entre histoire de la nature et histoire de l'homme. C'est l'affirmation de l'impossibilité de traiter ces histoires séparément. Serres dit dans le Contrat naturel ce que Dipesh Chakrabarty, l'historien postcolonial, dit presque quinze ans après. ${ }^{7}$ L'Anthropocène est le nom de la fin d'un partage qui organisait les connaissances entre les humanités et les sciences de la nature. Une des questions que les scientifiques se posent est maintenant de savoir s'il y a des critères pour assigner un début de l'Anthropocène. Ce n'est encore qu'un débat. Il faut qu'un congrès de géologie décide et pour ça il faut qu'il y ait des critères.

F.C. : Ne peut-on pas évoquer la Révolution Industrielle comme début ?

C.L. : C'est ce que tout le monde dit. Mais les chercheurs en géographie physique sont à la recherche de marqueurs stratigraphiques qu'on puisse repérer, qui soient à la fois repérables et globaux. Or la Révolution Industrielle, elle, ne se marque pas globalement ${ }^{8}$.

6 Michel Serres, Le Contrat naturel, Paris, François Bourin, 1990.

7 Dipesh Chakrabarty, "The Climate of History: Four Theses ", Critical Inquiry, vol. 35, n 2 (Winter 2009), p. 197-222.

8 Sur ces enjeux de datation de l'Anthropocène, cf. Simon L. Lewis et Mark A. Maslin, « Defining 
B.G. : En vous lisant, on aperçoit parfois cette idée que nous habitons le même monde qu'Aristote.

C.L. : Mais ça ne veut pas dire qu'on a complètement changé de monde.

B.G. : Du coup, l'Anthropocène ne serait-il pas moins le nom d'un nouveau monde que celui d'un régime de connaissance changé ?

C.L. : L'effort des géologues (géophysiciens, géographes, et stratigraphes), est de trouver des repères naturalistes. Ça ne veut pas dire qu'on vit différemment. Selon eux, le début d'une ère peut être marqué par l'arrivée d'une nouvelle espèce, mais ce n'est pas nous, la nouvelle espèce.

N.E.E. : N'y a-t-il pas du point de vue de la justice, quelque chose d'assez problématique dans l'usage même du concept d'Anthropocène ? Qui est en effet l'anthropos de l'Anthropocène?

C.L. : C'est ce qu'ont dit Christophe Bonneuil et Jean-Baptiste Fressoz. Ils ont proposé de l'appeler Anglocène en disant que ce n'est pas tous les hommes également qui sont à l'origine de cela. C'est le résultat du développement industriel occidental dont effectivement l'Angleterre ou la GrandeBretagne est l'aile marchante au XIX ${ }^{\mathrm{e}}$ siècle, dépassée ensuite par d'autres. Dans le volume qu'Émilie Hache a coédité, Du monde clos à l'univers infini (2014), Bonneuil reprend cette idée qu'il y a une dimension sociale et qu'on ne peut pas naturaliser les humains qui sont à l'origine des transformations industrielles qui conduisent à l'accumulation des gaz à effet de serre dans l'atmosphère. Je suis plutôt d'accord avec Bonneuil, à qui Chakrabarty répond en défendant la thèse selon laquelle l'Anthropocène veut dire qu'il ne faut pas seulement prendre l'humanité dans sa division sociale, mais qu'il faut aussi la prendre dans son unité d'espèce. Sa thèse est que l'Anthropocène n'est pas seulement la rencontre de deux histoires qu'on ne peut plus mélanger puisque l'humanité est devenue une force géologique, mais aussi qu'en devenant une force géologique elle doit être prise dans son unité naturelle spécifique. Ce que Bonneuil critique avec un certain nombre de bons arguments. Les questions de justice viennent à la fois de ce que la question écologique, la crise environnementale est un destin commun, quelque chose qui nous frappe tous, mais qui nous frappe de façon très inégale et dont nous sommes inégalement responsables. Par ailleurs, la question de la responsabilité est aussi l'objet d'affrontements violents, notamment chez les économistes, entre des gens comme Joan Martinez Alier, qui développe le concept de dette écologique (la dette écologique est le nom de la responsabilité des pays occidentaux visà-vis du reste du monde), et un certain nombre d'autres économistes comme Olivier Godard par exemple, qui soutient que cette responsabilité n'a pas lieu 
d'être, qu'elle n'existe pas. Pour Olivier Godard le concept de dette écologique n'est pas un concept rationnel mais une sorte d'archaïsme précontractualiste, une dette à la Mauss. Il y a donc un conflit violent. Tout le monde est d'accord sur le fait que le problème nous atteint tous. Mais sur la façon inégale dont nous sommes à la fois atteints et impliqués, et les conséquences que l'on peut en tirer, là il y a franchement débat.

N.E.E. : À propos de ce débat, est-il possible, comme s'interroge Axel Gosseries, de défendre l'idée d'obligation des descendants d'une génération de pollueurs à compenser les victimes actuelles des actions de leurs ancêtres sur la base de la notion morale de free riding transgénérationnel ?

C.L. : Cela devient un débat très technique. Pour moi ce n'est pas une affaire de compensation. C'est déjà réducteur de le dire. C'est ce que j'avais essayé de dire à Bordeaux ${ }^{9}$ mais ce n'était pas passé. C'est un problème de justice corrective et la justice corrective ne se dit pas dans les termes de la justice distributive. Quand vous commencez à parler de compensation, vous êtes déjà dans une vision distributive de la justice pénale. Ce n’est pas du tout évident. Et en plus, Gosseries va encore plus loin. Il dit que finalement il n’y a pas de responsabilité mais simplement free riding. C'est l'idée qu'on a profité gratuitement de quelque chose pour lequel les autres payaient. Ce n'est pas vrai. Pour qu'il y ait free riding, pour que vous soyez passagers clandestins dans un bus, il faut qu'il y ait un bus, il faut quelque chose de collectif. Il n'y a de free riding que dans une société organisée. Sinon on est dans la lutte de tous contre tous. Et de ce point de vue, la période coloniale n'est pas du free riding, mais de l'absence de justice. Donc, est-ce qu'il y a des compensations ? L'idée de la dette écologique ne relève pas d'une compensation. C'est l'idée de mettre en balance dette écologique et dette financière pour obtenir dans les négociations, une remise de la dette des pays du Tiers Monde ou du Sud (appelez les comme vous voulez). C'est vrai que tant que les pays en développement sont sous la pression extérieure de la dette, soit ils ne peuvent pas se développer, soit ils se développent en faisant du dégât environnemental. La vérité enfin trouvée du rapport Brundtland ${ }^{10}$, c'est de dire que tout va aller bien puisqu'une grande cause de la dégradation environnementale, c'est la pauvreté. Si on sort de la pauvreté, on sort de la dégradation environnementale. Laissons donc le Sud se développer. Cela implique qu'il faut que ces pays du Sud ne soient pas sous pression financière. Voilà l'idée de la dette écologique : obtenir qu'ils en aient

9 "Justice et Environnement : regards croisés entre la philosophie et l'économie ", Workshop organisé par le GREThA, Université Bordeaux IV, le 7 décembre 2012.

10 Texte de 1987 rédigé par la Commission mondiale sur l'environnement et le développement de l'ONU (présidée par Gro Harlem Brundtland), Our Common Future (Notre avenir commun) sous-tend le Sommet de la Terre à Rio de Janeiro (1992) et impose notamment la notion de "développement durable". 
un peu moins sur les épaules, qu'ils soient un peu moins contraints à surexploiter leurs ressources naturelles. Le traduire en demande de compensation, c'est déjà un affaiblissement. Et quand en plus on dit comme le fait Gosseries, qu'au fond cette vision pénale ce n'est pas ce qui fait marcher l'économie, on va tomber dans le free riding puisqu'il a un sens économiquement. L'exemple du free riding, c'est le phare. Le bateau profite de la lumière du phare, et il n'a pas forcément payé pour construire le phare. Quand il n'y a pas le marché, on demande à l'État d'intervenir. Les États vont prendre en charge, mais le problème de la dette écologique est beaucoup plus grave.

F.C. : Pour revenir au Bon usage de la nature, vous relevez l'idée que la modernité était travaillée par deux conceptions de la nature, la natura naturata issue de l'étude des corps inanimés et prévisibles, la natura naturans issue de l'étude des corps animés et imprévisibles. Dans quelle mesure l'écologie se rattache-elle à cette seconde conception et souligne ainsi du point de vue scientifique son appartenance à la modernité ?

C.L. : Ce n'est pas tout à fait ça que nous avons voulu dire avec natura naturata et natura naturans. La distinction est en latin parce qu' elle est scolastique, elle est reprise par Spinoza mais ce n'est pas lui qui l'invente. Pourquoi la reprenons-nous ? Parce que l'image que donne d'elle-même la modernité scientifique, c'est natura naturata et le mécanisme cartésien, ce que Voltaire énonce dans l'article Nature de son propre Dictionnaire philosophique : "On me dit nature mais je suis tout art». Cette idée que le monde est une machine est plus qu'une analogie. Comme il n'y a jamais de paradigme simple, ce qui est mis en avant est la mécanique, mais derrière se profile en continu la natura naturans, la physis grecque, en particulier en histoire naturelle. Les observations d'histoire naturelle sont le chemin de constitution du darwinisme, Darwin lui-même ayant fait son voyage en bateau. Sous une vision mécaniciste dominante, qui fait de la machine la métaphore clé (même pour Leibniz), demeure une autre vision de la nature comme processus en cours. Si l'écologie devient vraiment une science avec le concept d'écosystème, qui est encore lié aux équilibres de la thermodynamique (plus exactement dans la mécanique classique), maintenant avec Whitehead c'est l'idée de processus qui l'a emporté, même si l'idée mécanique demeure extrêmement puissante. Les rapports se sont inversés.

F.C. : Est-ce que la crise écologique ne démontre pas les limites du projet baconien, du projet mécaniste sur la vie, une mécompréhension de la vie ?

C.L. : Bien sûr. C'est pour ça qu'on peut être inquiet quand on entend les médecins se réjouir des cœurs artificiels.

F.C. : Ce qui veut dire que ce projet baconien atteint une totalité qui poserait des liens entre l'écologie et la bioéthique? 
C.L. : On peut dire ça. Mais il faut se souvenir aussi que Bacon écrit avant la mise en place de la philosophie mécaniste. Il n'a pas lu Descartes et ne connait pas Galilée, mais il met en place les cadres qui vont servir à penser le mécanisme. Carolyn Merchant a montré comment Bacon met en place tout son système avant même que ne se développe la philosophie mécaniste, qui est post-cartésienne ${ }^{11}$. C'est très intéressant de voir comment une sorte de modèle éthique est prêt chez Bacon alors que le contenu scientifique n'est pas encore là. Il y a beaucoup de métaphores organicistes chez lui (dans $L a$ nouvelle Atlantide en particulier). Il y a une fascination pour la naissance, la prolifération, le fait d'avoir des enfants, qui montre que la natura naturans est toujours là sous la natura naturata.

F.C. : En pensant à Haeckel, quels liens voyez-vous entre l'écologie et le vitalisme?

C.L. : Il y a une phase organiciste de l'écologie, dont je ne suis pas spécialiste. J'ai tendance à penser que l'écologie est nommée au XIX ${ }^{\mathrm{e}}$ siècle par Haeckel, mais démarre véritablement comme science au XXe siècle. L'écologie, c'est l'idée de l'interaction des organismes avec leurs milieux et des entités vivantes entre elles, ces interconnexions mais aussi ce à quoi elles aboutissent. Pour Frederic Clements, une série de successions où une végétation recouvre l'autre aboutit au climax conçu comme superorganisme. C'est contre cette vision organiciste qu'Arthur Tansley énonce son concept d'écosystème dans un remarquable article ("The Use and Abuse of Vegetational Concepts and Terms ", 1935) qui est une critique de l'écologie organiciste de Clements, où il précise qu'il emprunte le terme de Système au sens de système physique. C'est lui qui va faire basculer l'écologie de la biologie vers la physique et la thermodynamique (une physique dynamique), ce qui va lui permettre d'inclure l'inerte dans le milieu, sans perdre le lien avec la biologie. Les phases organicistes reviennent d'ailleurs. Worster et Callicott vous diront que la véritable opposition n'est pas tant entre Tansley et Clements mais entre Tansley et Clements d'une part et $\mathrm{H}$. A. Gleason de l'autre, un écologiste beaucoup plus individualiste. Holisme contre anti-holisme.

N.E. : En parlant d'interconnexion doit-on exclure de parler d'autonomie et d'hétéronomie de la nature?

C.L. : Quelle autonomie et quelle hétéronomie ? En quoi est-ce que la nature serait une hétéronomie?

N.E. : Quand on fait par exemple référence aux lois de la nature.

11 Carolyn Merchant, The Death of Nature: Women, Ecology and the Scientific Revolution, San Francisco, Harper \& Row, 1980. 
C.L. : L'écologie n'est pas une science de lois. La notion de loi en science est une notion positiviste qui ne vaut en gros que pour la science du $\mathrm{XIX}^{\mathrm{e}}$ siècle. Dans un numéro de la Revue de synthèse, nous avions fait une série d'ateliers sur l'histoire de la notion de $\operatorname{loi}^{12}$, dont le XIX siècle marque l'apogée, alors que dans les sciences contemporaines il n'y a plus de loi. Je n'ai jamais entendu parler de loi en écologie. Il y a des modèles, des variations statistiques. Épistémologiquement, la notion de loi a fait son temps. C'est Descartes qui parle de lois de la nature. Le nomos grec désigne uniquement les lois humaines. C'est pourquoi parler d'hétéronomie de la nature n'a pas de sens. Que des gens comme Luc Ferry puissent dire qu'aller chercher les normes dans la nature c'est une hétéronomie au sens kantien, ça n’a rien à voir avec les lois naturelles. C'est plutôt une analogie, très maîtrisée chez Kant.

B.G. : Ne peut-on pas parler comme Carl Schmitt de Nomos de la terre?

C.L. : On a le droit de faire des métaphores. Il y a un moment de rencontre entre ce qu'Althusser nomme les "lois-rapport " et les "lois-commandement ", même si je crois qu'il se trompe sur Montesquieu, mais c'est une rencontre très temporaire, dans laquelle nous ne sommes plus. Et vous savez à quel point la notion de loi en biologie est une notion très controversée. Sur les questions climatiques on n'est plus dans les lois mais dans les modèles, ce qui est tout autre chose. Peut-être que je me trompe mais en écologie, il n'y a pas de loi, seulement des modèles.

B.G. : Concernant l'articulation à laquelle vous tenez entre philosophie morale et philosophie physique, comment l'esthétique prend-elle place dans votre réflexion ? Vous semblez vous intéresser entre autres formes à l'écriture de la nature à la première personne, ou au land art par exemple.

C.L. : Land art et Ecological art sont deux choses très différentes. Je pense néanmoins que le sentiment de beauté que nous pouvons éprouver devant la nature est l'une des meilleures introductions à l'éthique environnementale.

B.G. : Un peu comme Humboldt écrivait que la contemplation du tableau d'un paysage était une invitation à l'étude de la nature ?

C.L. : Oui, sauf que ce n'est sans doute pas vrai. Je parlais bien du beau de la nature et pas du beau dans l'art. L'art est une création humaine et donc il n'y a pas de beau naturel. Nous avons tendance à ne trouver beau que ce qui peut être représenté, en lien avec la perspective. Ce n'est pas la nature, mais le paysage, le pittoresque. Est-ce qu'il y a un beau dans la nature ? C'est là toute la question. On connait dans la philosophie morale classique la proximité entre le goût esthétique et le goût en morale, et des analogies sont possibles.

12 Cf. Catherine Larrère, "Le gouvenement de la loi est-il un thème républicain ? ", Revue de synthèse, 2-3, 1997, p. 237-258. 
Si je m'intéresse à l'écologie à la première personne c'est qu'il y a des modes d'écriture qui permettent de dire des choses qu'un discours scientifique ne permet pas de dire. "Penser comme une montagne" ", ça fait rigoler si vous écrivez ça comme un essai de science, l'écrire littérairement permet de faire apparaître ce qui n’apparaît pas dans un essai scientifique.

N.E.E. : Pour revenir à la question de l'éthique environnementale et à celle de la justice, pensez-vous qu'on puisse élargir la communauté de justice comme on élargirait la communauté morale aux non-humains?

C.L. : Je ne pense pas. Il se développe dans les pays de langue anglaise dans les années 1970 une éthique environnementale qui est une réflexion sur les rapports entre l'homme et la nature et qui pose la question non seulement des devoirs moraux des hommes vis-à-vis de la nature, mais de l'inclusion de la nature dans la communauté morale. Est-ce que les entités naturelles ont un statut moral de la même façon qu'il va pour nous de soi, pour la philosophie morale dominante, que les êtres humains ont un statut moral ? Je pense qu'introduire les questions de justice environnementale, c'est passer de l'Homme (avec un grand $\mathrm{H}$ ) aux Hommes (au pluriel). Les questions de justice environnementale se posent quand, remettant les hommes dans la nature, on se rend compte de ce que tout le monde est atteint par les problèmes environnementaux, mais pas atteint également, et que tout le monde n'y contribue pas également. À partir du moment où vous passez de l'Homme aux Hommes, vous prenez en compte la pluralité des hommes, non seulement leur pluralité, leur diversité, mais aussi leur inégalité. Et à partir du moment où vous avez des questions d'inégalités, vous avez des questions de justice. Pour Aristote, le juste c'est l'égal. Quel égal ? Il y a quelque chose de profondément injuste dans le fait que les gens soient chassés de leurs îles dans le Pacifique parce que les eaux recouvrent leurs îles alors qu'ils n'y sont pour rien. De plus, chaque fois que vous avez une pluralité d'hommes, se présentent les problèmes de justice distributive. On peut dire d'une certaine façon que les questions de justice environnementale surgissent là où on oublie un peu l'éthique environnementale comme problématique de l'homme vis-à-vis de la nature. Une fois ces bases posées, un certain nombre de gens posent la question suivante : ces devoirs de justice n'existent-ils qu'à l'égard des hommes ou ne serait-ce pas aussi la justice à l'égard de la Terre, ou des animaux (puisque le changement climatique n'affecte pas que les humains, mais déjà quantité de non humains) ? Dans ce débat, certains comme Bryan Barry (reprenant la position de Hume, que Rawls reprend) disent que nous ne devons la justice qu'aux hommes, et qu'aux non humains nous pouvons devoir la pitié, la charité, la compassion mais pas la justice. D’autres, comme Dale Jamieson, David Schlosberg, disent

13 Cf. Aldo Leopold, Almanach d'un comté des sables, trad. Anna Gibson, Paris, Flammarion, 2000, p. 168-173. 
qu'il faudrait inclure les non humains dans les devoirs de justice. En fait ils ne s'y tiennent pas. Il est difficile d'y arriver. Certains prétendent que la justice environnementale est la distribution des biens et des aménités, des maux et des coûts environnementaux entre humains et que la justice écologique ne s'en tient pas aux humains, mais il faut voir comment ce qui est juste varie, d'autant que le right de Leopold n'est pas une question de fairness. Il y a déjà suffisamment de problèmes dans la définition de la justice environnementale entre humains, problèmes qui viennent de ce que l'environnement n'est pas un bien comme un autre qu'on peut distribuer. Selon moi c'est beaucoup plus en prenant en compte la spécificité des questions environnementales dans les questions de justice environnementale par rapport aux autres formes de justice distributive, qu'on répondra à comment se conduire bien dans la nature, plutôt qu'en ajoutant de façon formelle un siège supplémentaire qui serait celui des générations futures ou de la nature. Il y a des choses chez David Schlosberg qui m'intéressent beaucoup, mais pas sur le passage de l'environnemental à l'écologique. Sur la question de la justice environnementale, je ne suis pas d'accord avec Axel Gosseries. Ce n'est pas un cas parmi d'autres de justice distributive. Cela ne rentre pas dans les formes de justice distributive. C'est ce que disent Sholberg et Jamieson et là-dessus, je partage leur avis qui renvoie à Iris Marion Young. Il faut prendre en compte le fait que ce n'est pas un bien comme un autre, mais la nature. Je ne crois pas que l'on puisse ajouter simplement la nature parmi d'autres bénéficiaires d'un schéma formel.

N.E.E. : Que penser dès lors de la Constitution de l'Équateur qui intègre la nature dans la communauté de justice et des dilemmes pratiques de développement qui apparaissent lorsqu'il est question d'articuler protection de ces droits et développement?

C.L. : Je crois que les dilemmes pratiques de développement, comme vous dites, posent beaucoup plus la question de comment articuler des conceptions différentes de ce que nous appelons nature, donc davantage du côté de Descola, plutôt que de chercher à trafiquer du Rawls pour l'appliquer. Il y a un bon commentaire de Marie-Angèle Hermitte ${ }^{14}$ au sujet de la Constitution équatorienne. J'avais participé à un débat avec un représentant des indiens Quechua, partisan du Sumak Kawsay (buenvivir), avec Descola, et ça ne s'était pas bien passé, car cette Constitution est un hybride, là où Descola avait travaillé si je puis dire sur de l'authentique. En manière de trafic pour se faire reconnaitre à l'ONU et à l'Unesco, on injecte un peu d'occidental, et cela énerve les anthropologues. Il s'agit donc bien de questions de traduction. Il est question d'articuler des visions différentes et un certain nombre de visions qui n'ont pas le même rapport à la nature que nous. Tandis que si vous

14 Marie-Angèle Hermitte, "La nature, sujet de droit ? "Annales. Histoire, Sciences Sociales, 2011/1 (66 année), p. 173-212. 
faites comme Gosseries et prenez un schéma rawlsien, ce schéma a une vision dualiste classique de la nature : vous n'allez pas y arriver. C'est un travail un peu obscur de faire ce genre d'hybride, comme la Pachamama, mais c'est ainsi qu' on y arrivera.

B.G. : C'est donc une question qui engage les « humanités », une question littéraire, linguistique, textuelle : comment faire coexister des langues différentes dans l'écriture de nos devoirs vis-à-vis de la nature?

C.L. : Oui. On retombe sur les théories de Quine.

F.C. : J'aimerais aborder l'aspect politique puisque nous avons parlé d'inégalités d'impacts.

C.L. : Et d'inégalité des contributions. On ne reçoit pas les mêmes retombées, et on n'est pas de la même façon auteur des retombées. C'est la dette écologique. La dette écologique est une mesure non pas d'inégalités d'impacts, mais d'inégalités de contribution.

F.C. : Pouvez-vous commenter le fait que John Baird Callicott évoque une exception française de la philosophie de l'écologie et l'impute au fait que la philosophie politique en comparaison à la philosophie morale est plus prégnante en France qu'aux États-Unis? Y a-t-il selon vous en France une philosophie politique de l'écologie et quelles sont ses grandes lignes actuelles?

C.L. : Callicott commentait une lecture que j'étais allée présenter à Porto Alegre du Contrat Naturel de Michel Serres. Politiques de la nature (1999) de Bruno Latour est une lecture que je partage et qui rejoint ce que dit Serres : les problèmes environnementaux ne posent pas un problème de rapport entre l'homme et la nature, mais une question de redéfinition des rapports entre science et politique : pour reprendre le sous-titre du livre de Latour, la question est de savoir comment faire entrer les sciences en politique, et comment "régler l'affaire Galilée » (selon l'expression de Serres cette fois), cette séparation entre ce qui se dit de la nature et ce qui est dans la cité. Latour fait remarquer que cela conduit à donner une autorité, parfois démente, aux scientifiques. Ils sont ceux qui ont le droit, l'autorité de parler de la nature. Dans Politique de la nature, il s'interroge sur la question de savoir comment faire entrer les scientifiques en démocratie en mettant en cause l'autorité que bien malgré nous, nous leur avons donnée. Ce n'est pas le problème des Américains qui sont beaucoup plus scientistes que nous autres, les enfants d'Auguste Comte. D'autre part, quand on m'a nommée présidente de la fondation de l'écologie politique, j'ai rendu claire la distinction que j'avais faite entre ce que $\mathrm{j}$ 'appellerais les politiques écologiques et l'écologie politique. J'appelle "politique écologique " le fait (pas tout à fait nouveau mais d'une importance croissante) qu'il y a un certain nombre de politiques publiques qui ont en charge la protection de la nature, la prévention des risques, qui ont 
pris suffisamment d'importance pour qu'on y consacre un ministère. Quand Latour écrit Politique de la nature, c'est à cette politique écologique là qu'il pense, à la part que peuvent y prendre les politiques et les scientifiques, à la recomposition du rapport entre l'expert et le politique. "L'écologie politique " est quant à elle la tentative, l'ambition de construire un projet social différent, qui prenne en considération le fait que nous ne pouvons plus ignorer notre environnement. Cela renvoie à André Gorz, selon deux généalogies bien distinctes. Aux États-Unis, il n'y a ni l'un ni l'autre, le problème est différent. Il n'y a pour ainsi dire pas d'écologie politique. Les conditions d'une vie politique bipartite et l'histoire font qu'il n'y a pas de place pour cela. La protection de la nature y prend une tout autre place qu'en Europe. Callicott s'amuse sur l'exception française, mais il met le doigt sur quelque chose d'important : en France on ne peut pas faire sans politique.

F.C. : Concernant cette écologie politique telle qu' elle s'est développée en France, chez Gorz que vous avez cité, mais aussi chez Bertrand de Jouvenel, Jacques Ellul ou Bernard Charbonneau, est-ce que ce sont des traditions qui pour vous ont un poids, une importance, dans les enjeux de la philosophie de l'environnement en France ? Doit-elle se développer dans cette perspective d'une lecture politique de la crise écologique ou sociale?

C.L. : Bertrand de Jouvenel marque la première apparition de l'expression " écologie politique " mais en concurrence avec " économie politique ", ce qui atténue son importance. Est-ce que l'écologie politique est une nouvelle économie comme le pense Alain Lipietz ? J'ai fait ma thèse sur l'émergence de l'économie : on voit émerger au XVIII ${ }^{e}$ siècle en Europe, pas seulement une nouvelle science, mais une nouvelle façon de voir le monde, d'accorder de l'importance à ceux à qui on accordait aucune importance (Montesquieu lit ainsi Mandeville : un boutiquier devient subitement un citoyen respectable). On n'y pense plus dans l'économie, mais c'est ainsi qu'elle émerge comme recomposition de la vision du monde, passant des élites vers les travailleurs, ou "classes productives ", comme les appellent les Physiocrates par opposition à la fierté nobiliaire. Lisant cela, on peut se dire que l'écologie politique pourrait être cela, une nouvelle façon de voir le social. Quelque chose de cet ordre, chez Gorz, l'amène à faire une critique du marxisme, reprenant à Habermas le passage du social au monde vécu. Quand je discute avec Lipietz, pour qui j'ai beaucoup d'estime, j'ai l'impression qu'il pense ainsi. Mais en l'absence de parti « économiste ", peut-il y avoir un parti écologiste?

F.C. : Ce qui suppose des incompatibilités politiques en fonction de l'économie (sur l'échiquier politique) ?

C.L. : Mais tout le monde est libéral maintenant, en économie.

F.C. : Est-ce que l'écologie ne risque pas d'amener un dirigisme ? 
C.L. : C'est une question compliquée. Dominique Méda, dans La mystique de la croissance. Comment s'en libérer? (2013), montre qu'on peut aborder la question écologique de la façon dont au XIX ${ }^{\mathrm{e}}$ siècle on a abordé la question sociale, c'est-à-dire comme un déficit d'une approche purement individuelle, atomistique, d'individualisme méthodologique. Or vous savez comme moi que la découverte de la question sociale au XIX ${ }^{e}$ siècle, dans le socialisme révolutionnaire ou réformiste, s'est traduite par une demande d'État. C'est ce que laisse entendre, me semble-t-il, Dominique Méda. Dominique Bourg et Kerry Whiteside mettent beaucoup plus les pieds dans le plat, parlant de faillite de la main invisible et de faillite de l'ordre spontané de la pratique. Nous parlions tout à l'heure de free rider et de défaillance légitime du marché qui conduit à faire appel à l'État. À ce moment-là, peut-on dire qu'on va faire appel à l'État comme on a fait appel à l'État pour le social ? Honnêtement, je ne pense pas. D'une part, parce que la question sociale a des frontières et que la question écologique n'en a pas. On pouvait davantage prendre en charge les problèmes sociaux nationalement (ceux d'une classe ouvrière française " par exemple), mais écologiquement, on a bien essayé de faire arrêter le nuage de Tchernobyl aux frontières, mais en vain. Lécologie ignore les frontières et pose des problèmes globaux. Il y a peut-être une gouvernance, mais pas d'État mondial. D'autre part, à la différence de la question sociale, l'écologie ne débouche pas sur une demande d'État mais sur une demande de démocratie (participative, directe, etc.), et de prise en charge des choses.

N.E.E. : Dans Vers une démocratie écologique ${ }^{15}$, Dominique Bourg et Kerry Whiteside proposent de commencer à penser la démocratie écologique en se concentrant sur les gouvernements et les modes de représentation nationaux. Comment dès lors parvenir à penser la démocratie écologique au niveau mondial ?

C.L. : Au moins, personne ne souhaite un État mondial (qu'on appelait " monarchie universelle " jusqu’à la Révolution française). Montesquieu, repris par Kant, a montré que l'extension de l'aire politique conduisait au despotisme. Il y a des différences d'échelles. Ça ne veut pas dire qu'on ne peut pas démocratiser les institutions internationales. Vous savez comme moi que le terme de "gouvernance " est un terme extrêmement ambigu, recouvrant en fait l'économie. Il y a des choses qui ne peuvent être résolues qu'au niveau international et on n'y trouve pas les formes contraignantes de l'État. Plus on monte d'échelle, moins les institutions sont démocratiques. Les institutions nationales françaises, ou espagnoles, ou italiennes ne sont pas fabuleusement démocratiques, mais les institutions européennes le sont encore moins. Donc si vous montez encore d'une échelle, vous n'allez plus avoir de démocratie du

15 Dominique Bourg, Kerry Whiteside, Vers une démocratie écologique : le citoyen, le savant et le politique, Paris, Seuil, 2010. 
tout. C'est donc un énorme problème. Mais en même temps, il n'y a pas de solutions nationales aux questions écologiques.

B.G. : En descendant d'une échelle pour regarder ce qui localement se présente comme mouvement de défense d'un espace, les multiples zones à défendre $(\mathrm{ZAD})$ sont peu perçues comme démocratiques, dans le discours tenu sur elles de l'extérieur. N'y a-t-il pas là une mise hors-jeu?

C.L. : Mais il y a des gens qui vont vous dire que c'est là le grand laboratoire de la démocratie.

B.G. : Je ne suis pas loin de le penser.

C.L. : C'est pourquoi je disais que la question écologique et la question sociale ce n'est pas la même question. Ça se traduit par des formes nouvelles de démocratie et il y a énormément de choses qui se passent. Notre-Damedes-Landes ou Sivens attirent l'attention, entre beaucoup d'autres.

B.G. : N'est-ce pas là aussi une tradition moins visible de gens pour qui la perspective sociale révolutionnaire dès le $\mathrm{XIX}^{\mathrm{e}}$ siècle ne se traduisait pas par une demande d'État? Je pense à Élisée Reclus entre autres.

C.L. : Oui, mais ils ont été marginalisés. On les redécouvre maintenant.

B.G. : Ils sont intéressants dans cette redécouverte car ils permettent de dépasser autrement la binarité entre libéral et dirigiste.

C.L. : Tout à fait d'accord. Mais ce qu'apporte de nouveau la question écologique, c'est qu'elle échappe à la dualité État/marché. Si vous prenez par exemple Elinor Ostrom sur les Commons ${ }^{16}$, pour un certain nombre d'économistes elle n'apporte rien de nouveau mais elle est celle qui sort de la dualité État/marché. L’article de Garrett Hardin, "La tragédie des communs ${ }^{17}$ " tel qu'il apparaît, débouche sur l'alternative " ou bien on privatise, ou bien on fait appel à l'État ». Ostrom donne une solution qui n'est ni l'un, ni l'autre, mais repose sur la capacité d'autorégulation de groupes locaux. Certains objectent qu'au niveau international c'est inefficace. Reste que là où il y a de l'invention dans les questions écologiques, c'est dans des choses de ce genre. Si cela

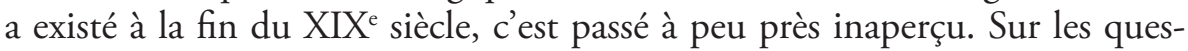
tions d'écologie politique, il y a quantité de constats, de difficultés, d'incompétences, d'impuissances relatives au fait de savoir comment faire au niveau international. Et en même temps, quantité de nouvelles choses émergent et se produisent, qu'il faut regarder pour agir et réfléchir.

16 Ostrom, Elinor. Governing the Commons: The Evolution of Institutions for Collective Action. Cambridge, UK: Cambridge University Press, 1990.

17 Garrett Hardin, "The Tragedy of the Commons ", in Science, vol. 162, 1968, p. 1243-1248. 
F.C. : La question de la marchandisation de la nature fait que l'écologie renvoie toujours aux questions d'économie. On a vu qu'une part importante de l'écologie se concevait comme une critique de l'économie politique issue du libéralisme. Y a-t-il des points de convergence critique entre l'écologie et ce qui a été la critique socialiste de l'économie politique?

C.L. : Qu'est-ce que vous appelez critique socialiste?

F.C. : Est-ce que l'écologie n'est pas dans l'obligation de parler de marchandisation de la nature, de recherche de profits, de rapports sociaux qui sont finalement très localisés (en Europe occidentale, dans le sillage de la Révolution Industrielle)?

C.L. : Non, il se passe plein de choses en Inde, en Afrique, en Amérique du Sud. Il y a beaucoup de choses qui se passent.

F.C. : Mais est-ce que ce n'est pas l'extension d'un système économique plus précisément né en Europe occidentale au XIX siècle, qui par le biais de la colonisation, s'étend et reproduit ce rapport à la nature à travers des rapports sociaux particuliers ? Finalement, le prolongement de la Révolution Industrielle et de l'économie de marché seraient-ils incompatibles avec la préservation de la nature?

C.L. : J'hésite à parler de crise écologique, parce qu'une crise a une solution, tandis que là il n'y en a plus, mais admettons. On peut dire que c'est l'aboutissement de quelque chose qui s'est mis en place au XIX ${ }^{\mathrm{e}}$ siècle avec la Révolution Industrielle, et la globalisation d'un certain type de rapport à la nature (sans beaucoup de résistance de la part de pays non-occidentaux, en pensant notamment au Japon). Si j'essaye de suivre votre raisonnement, on peut dire que cette industrialisation avait trouvé au XIXe siècle une critique socialiste, avec Marx et quelques autres. Le problème c'est que la réponse qui avait été proposée sous le nom de socialisme, consistait à partager et peut-être même à amplifier la même conception des rapports à la nature (ce qui avait amené des gens comme Raymond Aron à parler de "société industrielle ", pour passer par-dessus la distinction entre socialisme et capitalisme). S'il y a eu des désastres écologiques c'est certainement en Union Soviétique (la mer d'Aral). De ce point de vue-là, le socialisme du XIX ${ }^{e}$ siècle n'apporte rien. Mais à la limite, c'est plus ancien. J'ai eu la chance de discuter il y a quelques années avec Ignacy Sachs qui est un économiste qui avait quitté l'Allemagne au moment où Hitler est arrivé au pouvoir. Il a fait une partie de ses études en France. Un peu comme Albert Hirschman, il a fait des projets de développement pour l'Amérique du Sud. C'est Ignacy Sachs qui invente le " développement durable » de façon beaucoup plus radicale que ce qui sera ensuite reconnu dans le rapport Brundtland. Au lendemain de la Seconde Guerre mondiale, quand il va au Brésil et qu'Albert Hirschman va aussi en Amérique 
du Sud, les modes de développement qu'ils cherchent pour l'Amérique du Sud ne sont pas pris au capitalisme du Nord, ni dans les modèles socialistes. Avec l'URSS d'un côté, l'Europe et l'Amérique de l'autre, il y a aussi ce qu'on va appeler le Tiers Monde. Et l'idée d'Ignacy Sachs, l'un des instigateurs d'une réflexion extrêmement progressiste sur l'environnementalisme, c'est de dire que ce n'est pas le socialisme qui aidera à développer l'Amérique du Sud. C'est aussi l'idée que les solutions du capitalisme ne sont pas généralisables, et que le socialisme tel qu'il existe ne l'est pas non plus. Il faut donc inventer pour tout ce qui n'est pas directement inféodé à l'un des deux mondes, d'autres formes de développement. C'est là-dedans qu'on commence à avoir conscience des problèmes environnementaux. J'ajouterai à cela le terme de quart-monde, une expression que j'ai vu surgir en France entre la fin des années 1960 et le début des années 1970 pour parler des banlieues complètement défavorisées. C'est quand j'ai rencontré Callicott et les environnementalistes américains, que j'ai pris connaissance que par quart-monde ils désignaient les peuples autochtones qui n'étaient intégrables ni dans le capitalisme, ni dans le socialisme, ni dans ces formes corrompues qui se mettaient en place dans le Tiers-monde. C'est intéressant, cette convergence entre le questionnement environnemental, le souci de la nature, et la découverte de la non-universalité, ni du capitalisme, ni $\mathrm{du}$ socialisme. Le socialisme n'était pas une solution parce qu'au niveau environnemental il reprenait, en pire, l'espèce d'hybris, de démesure soviétique, mais aussi parce que ce n'était pas un mode de développement économique.

B.G. : Donc ce n'est pas une réponse, mais des réponses, un renoncement pluraliste à une ontologie ?

C.L. : Oui, un renoncement à un modèle unique.

N.E.E. : Face à ce quart-monde au sens de peuples autochtones, n'y a-t-il pas à leur égard une exigence de solidarité ? Il faut inventer de nouveaux modes de développement.

C.L. : Il faut qu'ils les inventent eux-mêmes. On ne va pas leur en apporter avec notre science. Ce n'est pas refuser la solidarité. Il s'agit de ne pas prendre une attitude paternaliste, de ne pas prétendre que nous saurions mieux qu' euxmêmes ce qui est bien pour eux.

N.E.E. : Mais ce n'est pas en ce sens là que je parle de solidarité. Ils font face à la crise environnementale, et la question n'est pas forcément à poser en termes de compensation à un niveau interétatique, mais plutôt en termes de relations avec eux. Plus précisément, comment les aide-t-on à s'adapter aux conséquences néfastes du changement climatique?

C.L. : Je n'ai pas de réponse dans l'abstrait. Il faudrait décliner cela sur des problèmes de biodiversité, par exemple. On ne les aidera pas en les mettant sous tutelle. Il faut donc voir en fonction de ce qu'ils demandent et de la 
façon dont ils voient les choses, de façon interactive. C'est autant d'études de cas. Comment ils sont affectés, en quoi cela change leurs vies, qu'est-ce qu'ils attendent. Mais il ne faut pas en faire des assistés.

F.C. : Quels sont d'après vous les enjeux majeurs de la philosophie de l'environnement pour le XXI ${ }^{e}$ siècle ? Est-ce que par exemple l'Anthropocène, la justice environnementale, l'écoféminisme selon vous vont changer la lecture?

C.L. : Je pense que ce qui avait marqué la fin du XXe siècle, c'est-à-dire la querelle autour de l'anthropocentrisme, est fini. Du moment où c'est global, on est dans l'anthropocentrisme. La distinction que fait Callicott est éclairante, entre anthropocentrisme moral (les humains ont en fait un statut moral au même titre si je puis dire que les autres bêtes, et sont touchés comme elles par le changement climatique global) et anthropocentrisme métaphysique (qu'il faut remettre en cause, selon lequel tout serait fait pour l'homme, qui serait au centre). Si on a pu penser qu'une certaine éthique environnementale était anti-humaniste, c'est fini. Pour moi, la querelle de l'anthropocentrisme est terminée, ce qui ne veut pas dire qu'elle ait été sans intérêt. Je pense qu'un grand apport des éthiques environnementales est de nous avoir sortis de notre chauvinisme humain, de notre solipsisme. De nous avoir fait comprendre que nous n'étions pas seuls au monde, que la nature existait. La ridicule fausse alternative entre « dernier homme ou dernier loup » n'a plus de sens du tout. Je pense que la question tourne désormais autour de l'unité et de la diversité de l'humanité. Une humanité inséparable de la Terre.

B.G. : Y a-t-il un point que vous auriez souhaité qu'on aborde avec vous?

C.L. : Si on n'a pas parlé d'écoféminisme, il était impliqué dans le questionnement de l'évidence de l'homme-nature. Et le fait que même s'il y a des analogies avec les combats sociaux du XVIII ${ }^{e}$ siècle, il y a des nouveautés. La question des femmes, c'est celle des nouveaux acteurs politiques émergents. Finalement les femmes ont mieux à faire que défendre leurs droits, donc elles se battent pour la nature.

Entretien mené, transcrit et présenté par :

Bertrand Guest

Maître de conférences Littérature générale et comparée Université d'Angers

Fabien Colombo

Diplômé de philosophie, économie-sociologie et anthropologie Université Toulouse Jean Jaurès

Nestor Engone Elloué Docteur en philosophie Université Bordeaux Montaigne 